\title{
New Method of Uncertainty Evaluation of the Sound Insulation of Partitions
}

\author{
W. BATKO* AND P. PAWLIK \\ AGH - University of Science and Technology, Faculty of Mechanical Engineering and Robotics \\ Department of Mechanics and Vibroacoustics, al. A. Mickiewicza 30, 30-059 Krakow, Poland
}

\begin{abstract}
The analysis of uncertainty related to evaluation of sound insulation of partitions performed according to standard solutions recommended in The Guide to the Expression of Uncertainty in Measurement (GUM) by ISO/IEC1998 and according to the new method based on the interval analysis - is presented in the paper. Both methods are compared and their application advantages presented. Limitations of the current solutions based on the law of propagation of uncertainty are shown. The method of the estimate derivation for the sound insulation based on interval arithmetic related to the measurement data is presented. The influence of the input parameters uncertainty on the calculation results of the sound insulation of partitions is determined. The considerations are illustrated by numerical examples.
\end{abstract}

DOI: $10.12693 /$ APhysPolA.123.1012

PACS: $43.55 .-\mathrm{n}, 43.55 .+\mathrm{p}$

\section{Introduction}

The basic task of investigations concerning acoustic properties of materials consists in obtaining reliable qualitative and quantitative information concerning their sound insulation. Its knowledge constitutes the grounds of the designing acoustic protections. Its erroneous evaluation can lead to wrong choices of design solutions. Thus, the assessment of the sound insulation uncertainty is a very important element for calibration laboratories, their clients, and users utilizing these results in the design process. Uncertainty is inseparably related to the measurement results. All kinds of estimations deprived of uncertainty evaluations are, in fact, only recommendations.

The assessment result of the sound insulation depends on the applied research procedure and accuracy of measuring instruments, as well as on the competence of persons performing measurements. They bring some uncertainty element into the obtained result. The basic, generally used solution in the domain of measurements uncertainty evaluation, is the methodology presented in the classic international document, The Guide to the Expression of Uncertainty in Measurement (GUM) [1]. Procedures described in the Guide are accepted by the Weight and Measured Offices and are obligatory in their accredited laboratories. They became an official tool of the uncertainty evaluation of given parameter measurement results. Their basic solution is an application of the law of propagation of uncertainty in the estimation of the measurement result. The application range of this solution concerns measurement observations, which can be treated as independent realizations of random vari-

*corresponding author; e-mail: batko@agh.edu.pl ables of a normal distribution, disregarding their time and space relations.

However, recommendations of GUM [1] within the scope of the type A measurements uncertainty evaluation do not provide information in what way this evaluation should be performed when changes of the measured parameters are not of a normal distribution. There are also no recommendations concerning verification of assumptions related to the method when the research practice limits the number of measurement results. It is even possible to claim that in case of acoustic investigations related to the estimation of acoustic parameters of materials, provisions in force not fully cope with limitations of the research practice and applied assumptions are often not verifiable.

Therefore an attempt was made to develop an approach of the acceptable mathematical formalism, in which the idea of errors propagation would be maintained with simultaneous giving up the assumptions limiting its application. The authors consider usefulness of connecting the proposed approach with the interval arithmetic formalism [2, 3]. It is based on assigning to measuring variables the determined interval of their variability, identified in a limited (in the statistical analysis sense) measuring test. Using variability intervals in calculations of the estimated parameter - according to the interval arithmetic formalism - allows to determine the possible error range for this parameter.

\section{Measurements of the sound insulation}

Measurements of the sound insulation of building partitions is carried out in the laboratory equipped with coupled reverberation chambers where the tested partition is placed between the chambers. The specific sound insulation is determined on the basis of measuring the sound pressure level in the sending and receiving chambers in 
several measuring points and on the basis of the reverberation time measured in the receiving chamber. The sound insulation is described by the formula:

$$
R=L_{1}-L_{2}+10 \log \frac{S T}{0.16 V},
$$

where $L_{1}$ is the average sound pressure level in the sending chamber, $L_{2}$ - the average sound pressure level in the receiving chamber, $S$ - the sample surface area, $V$ the receiving chamber volume, $T$ - the receiving chamber reverberation time.

Parameters of the sound pressure level $L_{1}$ and $L_{2}$ and the reverberation time $T$ have been measured in onethird-octave bands, in 70 measuring points, in order to estimate the uncertainty of these parameters. Two-channel frequency analyser Norsonic RTA 840 was used in the measurements. The measurement has been performed for the polycarbonate plate of dimensions $2 \times 1 \times 0.02 \mathrm{~m}^{3}$ in the laboratory of the coupled reverberation chambers where the volume of the sending chamber was $178.77 \mathrm{~m}^{3}$, while the receiving chamber volume was $176.9 \mathrm{~m}^{3}$.

\section{Determination of variability intervals of input parameters}

On the basis of the 70-element measuring test, the variability intervals of individual parameters have been determined as a frequency function with a confidence level equal to 0.95 . The percentile method was used for this purpose. Percentiles of the order 2.5 and 97.5 have been determined to be able to find the endpoints of the variability intervals of parameters $L_{1}, L_{2}$ and $T$. Lower and upper interval limits as a frequency function are presented below in Figs. 1-3.

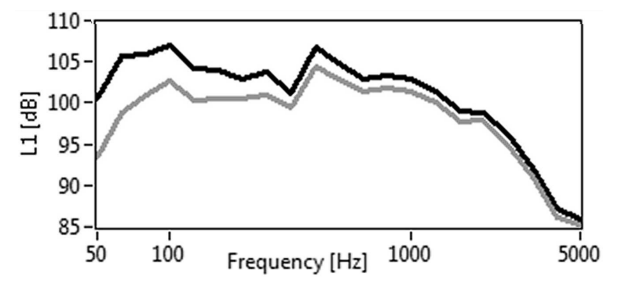

Fig. 1. Variability interval of $L_{1}$ parameter as a frequency function, with a confidence level $1-\alpha=0.95$. Grey curve - lower limit, black curve - upper limit of the interval.

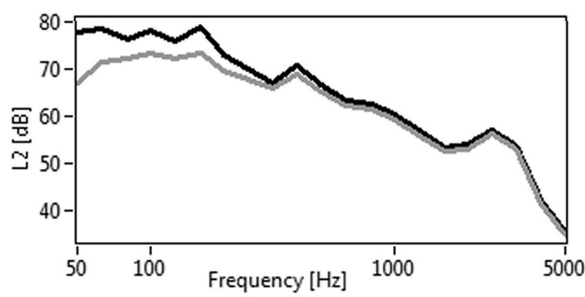

Fig. 2. Variability interval of $L_{2}$ parameter as a frequency function, with a confidence level: $1-\alpha=0.95$. Grey curve - lower limit, black curve - upper limit of the interval.

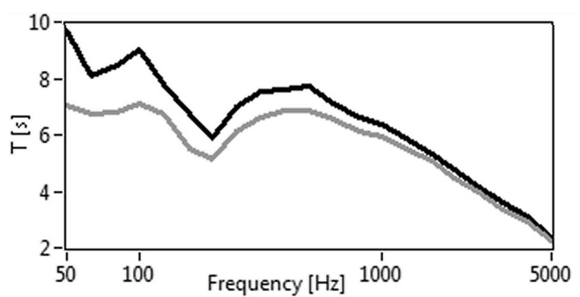

Fig. 3. Variability interval of $T$ parameter as a frequency function, with a confidence level: $1-\alpha=0.95$. Grey curve - lower limit, black curve - upper limit of the interval.

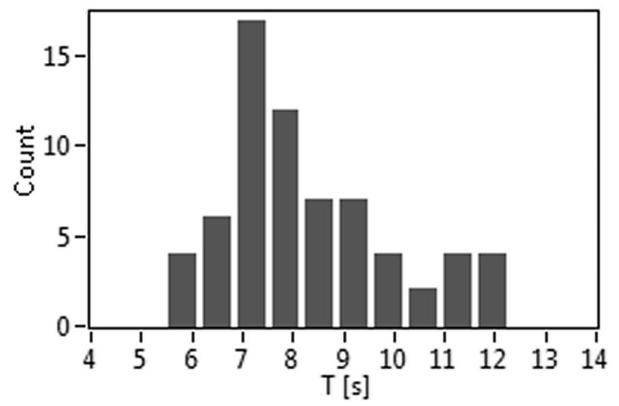

Fig. 4. Histogram of $T$ parameter for frequency $50 \mathrm{~Hz}$.

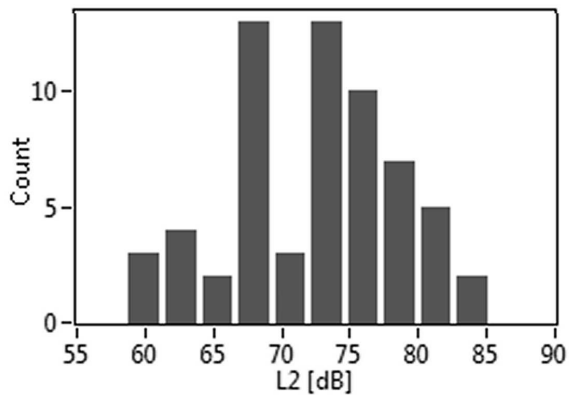

Fig. 5. Histogram of $L_{2}$ parameter for frequency 50 $\mathrm{Hz}$.

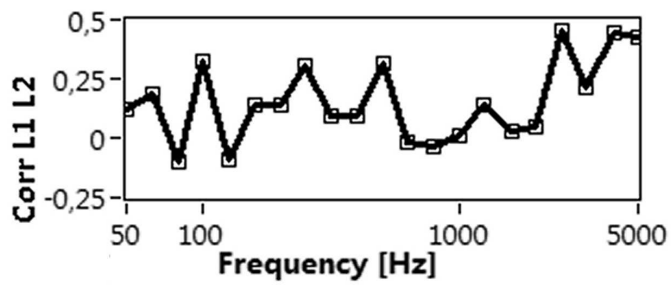

Fig. 6. Correlation coefficient between sets of parameters $L_{1}$ and $L_{2}$ as a frequency function.

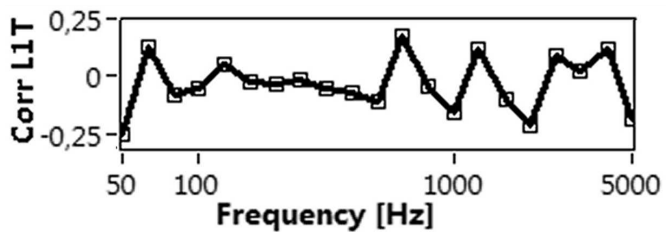

Fig. 7. Correlation coefficient between sets of parameters $L_{1}$ and $T$ as a frequency function. 


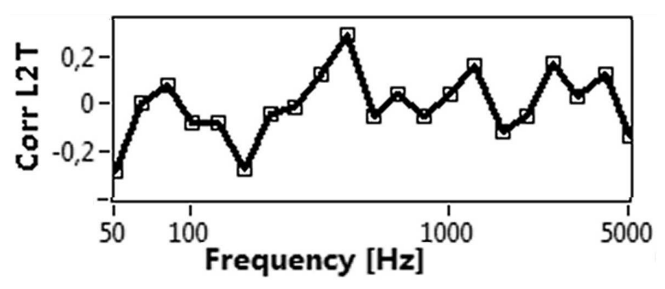

Fig. 8. Correlation coefficient between sets of parameters $L_{2}$ and $T$ as a frequency function.

The percentile method was used for the confidence level determination due to the fact that distributions of acoustic parameters were asymmetric (Figs. 4, 5) which not allow to apply the law of propagation of uncertainty. A similar problem of the confidence level determination for asymmetric distributions was considered in the paper of Cox and Marris [4].

Then, the correlation coefficients between sets of input parameters have been determined (Figs. 6-8).

\section{Uncertainty evaluation using interval arithmetic}

The formalism describing operations on the intervals started in the 1950s. It was named the interval arithmetic in the 1960s in the papers of Moore [2, 3] and was used for controlling round-off errors in numerical calculations. In the following years, several expansions of the classic interval arithmetic were created due to, among other things, a tendency of over-estimation of resulting intervals. The method of reducing intervals on the basis of the correlation coefficient between sets of errors was created. This method was proposed by Jakubiec [5] and named the reductive interval arithmetic (RIA). The advantage of the interval analysis is the uncertainty control at each stage of calculations, since all mathematical operations are carried out in the interval arithmetic formalism. This property significantly simplifies an implementation of this method in numerical devices. It is enough to implement the basic operations of the mathematical calculation formalism, and then perform mathematical operations according to this formalism.

In the present paper RIA is applied for determining uncertainty in modeling the sound insulation. Utilizing the confidence intervals determined in Sect. 3, the initial parameters $L_{1}, L_{2}$, and $T$ written in the form of interval numbers defined as follows:

$$
\boldsymbol{x}=[\underline{x}, \bar{x}]=\{x: \underline{x} \leq x \leq \bar{x}\},
$$

where $\underline{x}$ is the lower interval limit - infimum, while $\bar{x}$ denotes the upper limit - supremum, and $x$ is an arbitrary real number belonging to the interval. The formalism of RIA was described in the paper of Jakubiec [5]. This reductive interval analysis was performed by means of the library in the calculation environment LabVIEW developed specially for this purpose. The basic operations on interval numbers necessary for the sound insulation determination was implemented.
The results of the reductive interval analysis are presented below in Fig. 9. The upper curve represents the upper limit of the variability interval of the sound insulation as a frequency function, while the lower curve shows the lower limit of the variability interval as a frequency function.

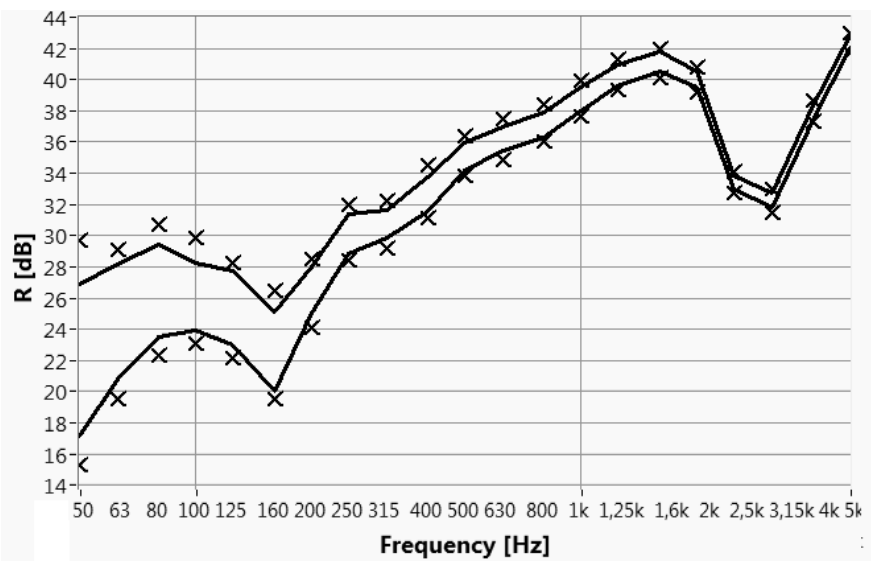

Fig. 9. Interval of the sound insulation $R$ as a frequency function, with the confidence level: $1-\alpha=0.95$. Black crosses - law of propagation of uncertainty, black lines - reductive interval analysis.

\section{Uncertainty evaluation using law of propagation of uncertainty}

In order to compare the functionality of uncertainty evaluation methods, the uncertainty analysis has been performed by means of the law of propagation of uncertainty with taking into account the correlation between sets of parameters. In case of extended calculation models, this method is difficult to implementat on numerical devices due to the necessity to estimate partial derivatives. It has also limitations on account of distributions of the set of input parameters.

Using the law of propagation of uncertainty (3), the total uncertainty of the input insulating power can be estimated. Uncertainties of input parameters are determined on the basis of the expected value and standard deviation:

$$
\begin{aligned}
& u_{c}(y)=\left[\sum_{i=1}^{n}\left(\frac{\partial f}{\partial x_{i}}\right)^{2} u^{2}\left(x_{i}\right)\right. \\
& \left.+2 \sum_{i=1}^{n-1} \sum_{j=i+1}^{n}\left(\frac{\partial f}{\partial x_{i}}\right)\left(\frac{\partial f}{\partial x_{j}}\right) u\left(x_{i}\right) u\left(x_{j}\right) r\left(x_{i}, x_{j}\right)\right]^{\frac{1}{2}},
\end{aligned}
$$

where $u\left(x_{i}\right)$ is the uncertainty of the i-th input parameter, $\frac{\partial f}{\partial x_{i}}$ - partial derivative of the function of the i-th parameter, and $r\left(x_{i}, x_{j}\right)$ - the correlation coefficient between the $\mathrm{i}$-th and $\mathrm{j}$-th parameter.

The results of the uncertainty analysis, determined by means of the law of propagation of uncertainty and by means of RIA are presented in Fig. 9. 


\section{Final remarks}

When determining the evaluation of the measurement result of the sound insulation of partitions in the laboratory practice, as well as assessing its uncertainty, there is a lack of practical possibilities of a reliable verification of assumptions of statistical method of the evaluation type A uncertainty. The inaccurate set of assumptions forces searching for the alternative method of the uncertainty evaluation. One of the propositions is considered in the present paper.

Its realization is connected with the interval arithmetic formalism basic operations of which were illustrated by the considered problem. The presented dependencies enabled, on the basis of the measurement results of initial values and their observed scatter, to calculate the possible variability range of the estimated parameter, i.e. uncertainties of the expected values of the sound insulation. The investigation results of the presented unconventional evaluation of uncertainty of the partitions sound insulation in relation to the results obtained by the statistic type A procedure determined by GUM [1] are given in the paper. A good convergence of the assessment results and easiness of realization make the proposed method worth recommendation. In particular, it loosens assumptions adopted in traditional calculation procedures which makes it more suitable for the labo- ratory practice. Propositions of using its suitability in various applications are presented in other publications of the authors $[6,7]$.

The proposed methodology of uncertainty evaluation and the obtained results indicate its methodological correctness. It can be also applied at working out the results of inter-laboratory comparisons.

\section{References}

[1] ISO/IEC, Guide to the expression of uncertainty in measurement, 1998.

[2] R.E. Moore, Ph.D. Thesis, Stanford University, Stanford 1962.

[3] R.E. Moore, Interval Analysis, Prentice-Hall, Englewood Cliffs 1966.

[4] M.G. Cox, P.M. Harris, Software Support for Metrology Best Practice Guide No. 6, Uncertainty Evaluation, National Physical Laboratory, Teddington 2006.

[5] J. Jakubiec, Application of Reductive Interval Arithmetic to Uncertainty Evaluation of Measurement Data Processing Algorithms, Silesian University of Technology Press, Gliwice 2002, p. 23.

[6] W. Batko, P. Pawlik, Archiv. Acoust. 37, 57 (2012).

[7] W. Batko, P. Pawlik, Acta Phys. Pol. A 121, A-152 (2012). 